\title{
Mitral Valve Abnormality Detection Using Deep Learning Approach in PLAX view in TTE
}

\author{
Vishal Chandra ${ }^{1}$, Prattay Guha Sarkar ${ }^{2}$, Vinay Singh ${ }^{3}$ \\ ${ }^{1}$ Usha Martin University, India, vcvishalchandra@gmail.com \\ ${ }^{2}$ Rajendra Institute Of Medical Sciences, India, prattay_2904@yahoo.com \\ ${ }^{3}$ Usha Martin University, India, vinaysinghuma@gmail.com
}

\begin{abstract}
To identify an abnormality in mitral leaflets is the main objective of this research automatically using transthoracic echocardiography with the help of deep learning techniques. underdeveloped countries are still under the burden of valvular diseases, mainly mitral valve disease, which is around 80 percent contribution in valvular diseases. assessments of mitral valve disease are totally based upon the morphology of mitral leaflets according to World Heart Foundation guidelines. Assessment is always subject to argument, due to its dependency on the echocardiographer's experience. Measurement of the pliability of leaflets, the thickness of leaflets, calcification detection, required highly experienced echocardiographers which have not only sound knowledge of echocardiography and but also morphology. our proposed deep learning model successfully able to classify between normal and abnormal mitral valve with accuracy $98 \%$, If an abnormality in mitral leaflets detected then further investigation required otherwise there is no investigation needed, measuring thickness, mitral valve area not be required to measure for the assessment of mitral valve. This research consists of two phases the first phase automatically locates the region of interest second phase classifies the mitral leaflets they are normal or abnormal. For locating the yolo3 model mechanism with dilated darknet53 is used for taking the area of interest automatically and for classification of normal and abnormal mitral leaflets, for classification depthwise convolution is used, the proposed pipeline is the combination of both, having accuracy $=99 \%, \mathrm{mAp}=86$ score, and other matrices have measured. PR and ROC curves are drowned to support the results in the evaluation. The motive of this research is to serve nonexpert to identify abnormalities in mitral leaflets. We used the Parasternal Long Axis (PLAX) for this research.
\end{abstract}

Key words: Mitral valve detection, Object detection, abnormalities, classification.

\section{INTRODUCTION}

Rheumatic heart diseases (RHD) are responsible for abnormalities in mitral leaflets in 99 percent of cases. In developing countries, RHD is vanished due to proper medicine, hygiene food, and a good lifestyle. Acute rheumatic fever, which is due to the infection of streptococcal pharyngitis, is responsible for Rheumatic heart disease (RHD). in developed and underdeveloped countries RHD is still a major burden in these countries, for cardiovascular morbidity and mortality, RHD is responsible for patients [1]. RHD affects mitral valve by mitral regurgitation (MR), Mitral mixed lesions and Mitral Stenosis (MS), results in heart failure. 2D echocardiography is the easiest and cheapest technology for the detection and diagnosis of RHD. RHD spawn globally in past 25-years [2], $34 \times 10^{6} \mathrm{RHD}$ patients have lost they're of life years $9.5 \times 10^{6}$ and it was estimated 323500 death alone in 2015 due to RHD. Although these estimations were drastically fallen since data unavailability of fewer regions across the globe, not inclusion and misdiagnosis of subclinical RHD in the study may lead to death. As studies say we must not unnoticed these facts [3], in subclinical disease clinical RHD exists in every 3 out of 10 cases, which cannot be ignored completely. patients with the early stage in RHD proper treatment is possible without much compromising with the health situations. since the easy availability of portable echocardiography machines, there is drastically increase in the rate of RHD identification, assessment of RHD with the help of echocardiography modality WHF (World Heart Federation) has proposed guidelines [4]. heart muscles, valve leaflets mainly mitral valve leaflets are affected due to RHD. Assessment of RHD is entirely based upon the morphology and function of mitral leaflets as per WHF guidelines for echocardiography. identifying the mobility and morphology Brightness mode (B mode) is used of mitral leaflets in echocardiography view. In this research we chosen the Parasternal Long Axis (PLAX) view of the determination of abnormal and normal leaflets are shown in Fig. 1a and $1 \mathrm{~b}$.

Inter-observer variability for the assessment of mitral valve is very high there for assessment is based upon AMVL (Anterior Mitral Valve Leaflet) instead of PMVL (Posterior Mitral Valve Leaflet [4], which is easily visible in PLAX view, this the reason why we have chosen PLAX view for this research. Studies say the mitral leaflet tip is the most affected part of the whole mitral valve [5]. In under developing and undeveloped country assessment, it is very difficult to manage time and operator's experience. the goal of this research to minimize the operator's dependency in screening settings, and provide help for operators at first sight and to reduce subjectivity, improvement in diagnosis. images obtained from echocardiography are highly affected by shadows, attenuation, and speckles. these images are also affected 
by the incorrect gain setting and individual sonographer which may lead to the incorrect diagnosis. in this research, the authors proposed a pipeline that automatically classifies the abnormalities and normal in mitral leaflets in PLAX view. rheumatic, degenerative and congenital are the cases when mitral leaflets are deformed and abnormality exists. In this research, we only used rheumatic heart disease and normal patient's echocardiography images and clips. in figure $1 \mathrm{a}$ and $1 \mathrm{~b}$.

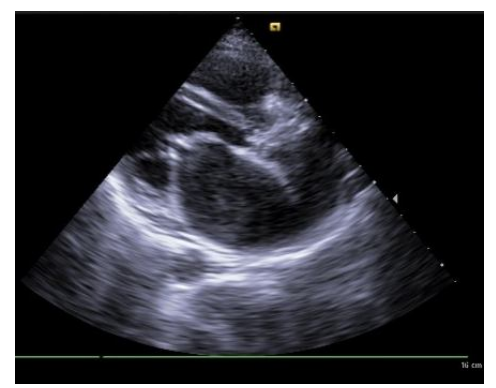

Figure 1a:Abnormal Leaflets

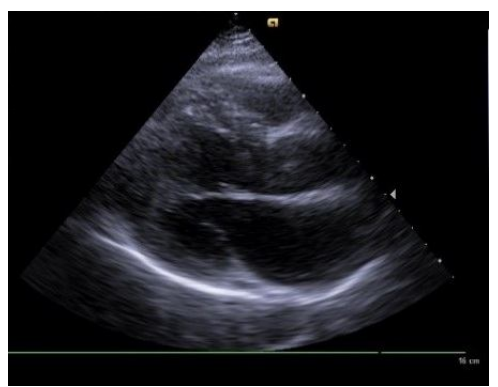

Figure1b: Normal Mitral Leaflets

\section{LITERATURE SURVEY}

In the last five years, various researches have done on object localization, detection, and classification of objects in space, time or both. [9] authors proposed a CNNs model for 2D MRI slices, identifying landmarks on the distal femur surface.[10] They reformulated the same problem for the localization of regions of interest (ROIs) around anatomical regions (aortic, descending aorta and heart) in a 3D CT volume 3D bounding box. [11] authors reformulated the problem and used pre-trained CNNs using RBM. [12] Gaussian representation with CNNs used by authors for landmark locations. for detection of landmark reinforcement learning used by authors [13] for the detection bifurcation of carotid artery decomposed 3D convolution for reducing the complexity of the model [14] used the method that decomposed 3D convolution with three one-dimensional CNN used. deep learning method called sparse adaptive, used by authors for the detection of aortic valve [13]. Long short-term memory used with CNNs in mid-pregnancy for detection of 12 standard planes [14] in fetal ultrasound. authors used CNNs in cinMRI for the detection of systolic and [15] diastolic movement of the heart. internal bleeding detection authors multi-stream CNNs integrating CT and PET [16] in the brain[18].The authors used a weakly supervised learning method for the detection of lesions in chest radiography and mammography identifying nodules[19]. [20] object detection method inspired by RCNN used for the detection of thyroid papillary cancer in thyroid ultrasound. Authors reformulation the same problem has done [21]. [22] authors used the inceptionv3 model for the detection of thyroid nodules in ultrasound.[23] for the detection of regional wall motion abnormalities comparative study with the existing model used. [24] 3D CNNs used by authors used for the classification of ejection fraction. [25] for echo view classification they used CNNs with 15 standards for different echo view classification.

\section{DATA COLLECTION AND ANNOTATION}

Samples were collected from COSY CARE hospital, Ranchi, Jharkhand, India under informed consent of all subjects. A total of 60 clips have taken from 60 different patients with 20 RHD and 40 Normal people with age from 15-70 years. Total 1800 images obtained by taking during the beginning of systole, mid systole and endsystole of the left ventricle to capture mitral leaflets thus 1800 images chosen for training and 40 for testing with 1169 images. Data labeled by experienced sonographers using labelimg [26] tool which is open source available on GitHub. Interobserver variability was insignificant by observing their opening width and the length of leaflets. Frames of mitral leaflets are also classified by an experienced sonographer to create the dataset.

\section{DILATED YOLO3}

We use dilation in Darknet53 in convolution layers and used the YOLOv3 mechanism for locating the area of interest which is Mitral Valve location in PLAX view. The name YOLO refers to "You only look once" as the name given by their author, due to low inference time it faster as compares to another object detection model like SSD, RetinaNet, RCNN. since it has low inference time and without losing much accuracy, it can localize, that is why we have chosen it. due to DarkNet53, it us better, stronger but not faster than YOLO2, DarkNet53 makes it complex but more accurate than YOLO2. The major problem with YOLO2 was it unable to detect smaller objects that were overcome in YOLO3. DarkNet53 is used in YOLO3 as shown in figure3, having 53 layers, FPN like architecture. In this research, we used same DarkNet53 with dialation of 2 in all convolution layer. this increases the mAP 1.5. 


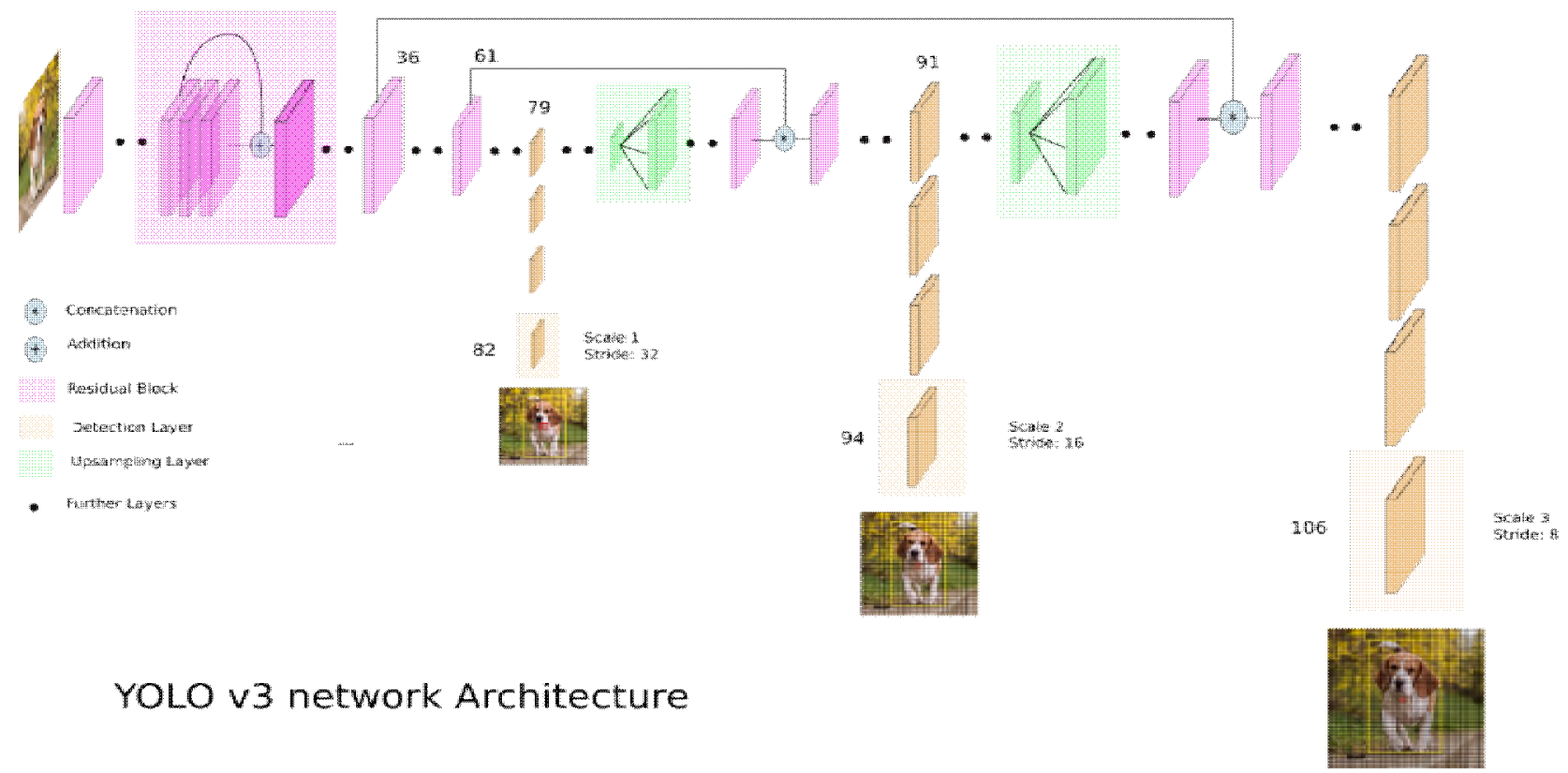

Figure 2: YOLO3 DarkNet53

Source Image Credit - https://towardsdatascience.com/yolov3-object-detection-53fb7d 3bfe6b

Figure 2 shows yolo3 object detection mechanism on three different scales which is salient feature of it. YOLOv3 is an FCN and 1 x 1 kernel as output of the feature map. In YOLO v3, with three different sizes and places it uses for the $1 \times 1$ detection kernels on feature maps for detection process.

Fundamentally input image is divided into $\mathrm{S} \times \mathrm{S}$ grid cells in YOLO, one and only one object is predicted by every grid cell. In each grid cell,

- $\quad$ confidence score and the number of bounding boxes is B that can be predicted.

- one and only one object is detected regardless of any number of predicted bounding boxes,

- conditional probability is given to each and every box.

DarkNet53 uses 18.57 billion parameters while our model has 61,576,342 parameters. Yolo3 does predictions at distinct scales.

1. The feature map layer is the last layer.

2. Goes to two-layer back and it upsampled twice, besides the higher resolution feature map is also taken and element-wise addition is done with merged upsampled feature map and application of convolutional filters on the merged map is done that results to the second prediction.

3. By repeating the two procedures we got the higher-level semantic structure of the feature map layer and results in the object location with better spatial resolution information.

YOLOv3 uses 9 anchor boxes. For each scale, there are three. The structure of dilated DarkNet53 is shown in figure 3 used for this research.

\begin{tabular}{|c|c|c|c|c|}
\hline & Type & Filters & Size & Output \\
\hline & Convolutional & 32 & $3 \times 3$ & $256 \times 256$ \\
\hline & Convolutional & 64 & $3 \times 3 / 2$ & $128 \times 128$ \\
\hline & Convolutional & 32 & $1 \times 1$ & \\
\hline \multirow[t]{4}{*}{$1 \times$} & Convolutional & 64 & $3 \times 3$ & \\
\hline & Residual & & & $128 \times 128$ \\
\hline & Convolutional & 128 & $3 \times 3 / 2$ & $64 \times 64$ \\
\hline & Convolutional & 64 & $1 \times 1$ & \\
\hline \multirow[t]{3}{*}{$2 \times$} & $\begin{array}{l}\text { Convolutional } \\
\text { Residual }\end{array}$ & 128 & $3 \times 3$ & $64 \times 64$ \\
\hline & Convolutional & 256 & $3 \times 3 / 2$ & $32 \times 32$ \\
\hline & Convolutional & 128 & $1 \times 1$ & \\
\hline \multirow[t]{3}{*}{$8 \times$} & $\begin{array}{l}\text { Convolutional } \\
\text { Residual }\end{array}$ & 256 & $3 \times 3$ & $32 \times 32$ \\
\hline & Convolutional & 512 & $3 \times 3 / 2$ & $16 \times 16$ \\
\hline & Convolutional & 256 & $1 \times 1$ & \\
\hline \multirow[t]{3}{*}{$8 \times$} & $\begin{array}{l}\text { Convolutional } \\
\text { Residual }\end{array}$ & 512 & $3 \times 3$ & $16 \times 16$ \\
\hline & Convolutional & 1024 & $3 \times 3 / 2$ & $8 \times 8$ \\
\hline & Convolutional & 512 & $1 \times 1$ & \\
\hline \multirow[t]{3}{*}{$4 \times$} & $\begin{array}{l}\text { Convolutional } \\
\text { Residual }\end{array}$ & 1024 & $3 \times 3$ & $8 \times 8$ \\
\hline & Avgpool & & Global & \\
\hline & Connected & & 1000 & \\
\hline
\end{tabular}

Figure 3: DarkNet53 Dilated 


\section{PROPOSED CLASSIFICATION MODEL}

Models consist of 19 having 8 convolutional layers, 4

MaxPooling.one Flatten, 2 dense and 2 dropout layers with

1 Dense layer figure 4.

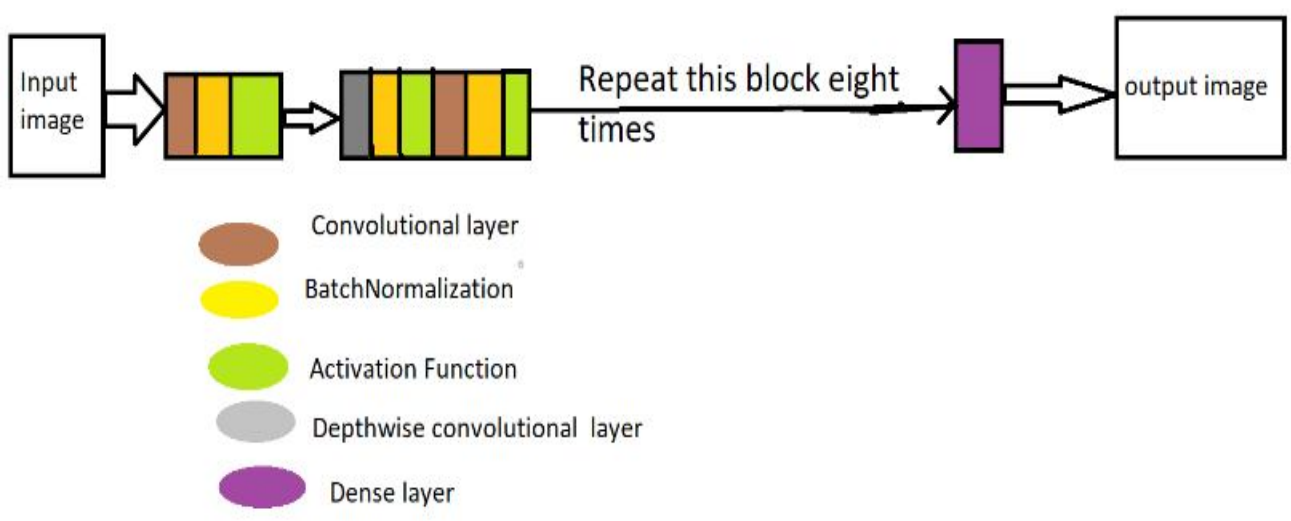

Figure 4:Proposed Model for Classification

Description of the proposed classification model

- First block convolutional layer $(32,3,2)$, BatchNormalization and ReLu.

Identity block



* Second block BatchNormalization.

* Second block convolutional layer $(32,3,2)$.

* second block Batchnormalization.

* second block ReLu.

Identity block is repeated 8 times with multiplying filter size by 2 each time.

\section{PROPOSED WORK}

Whole work is combination of two parts first automatic detection of the mitral valve region in PLAX view which is our region of interest by dilated yolo3, it provides mitral leaflets bounded area which is used in second part of pipeline,the second part is a MobileNet inspired depthwise convolutional technique used model, the proposed model classify mitral leaflets whether they are normal or abnormal. The whole system works end to end automatically, no human or manual intervention required. The whole pipeline is shown in figure 5. depthwise convolution has a smaller number of parameters.

- 1 Dense layer have 1. 


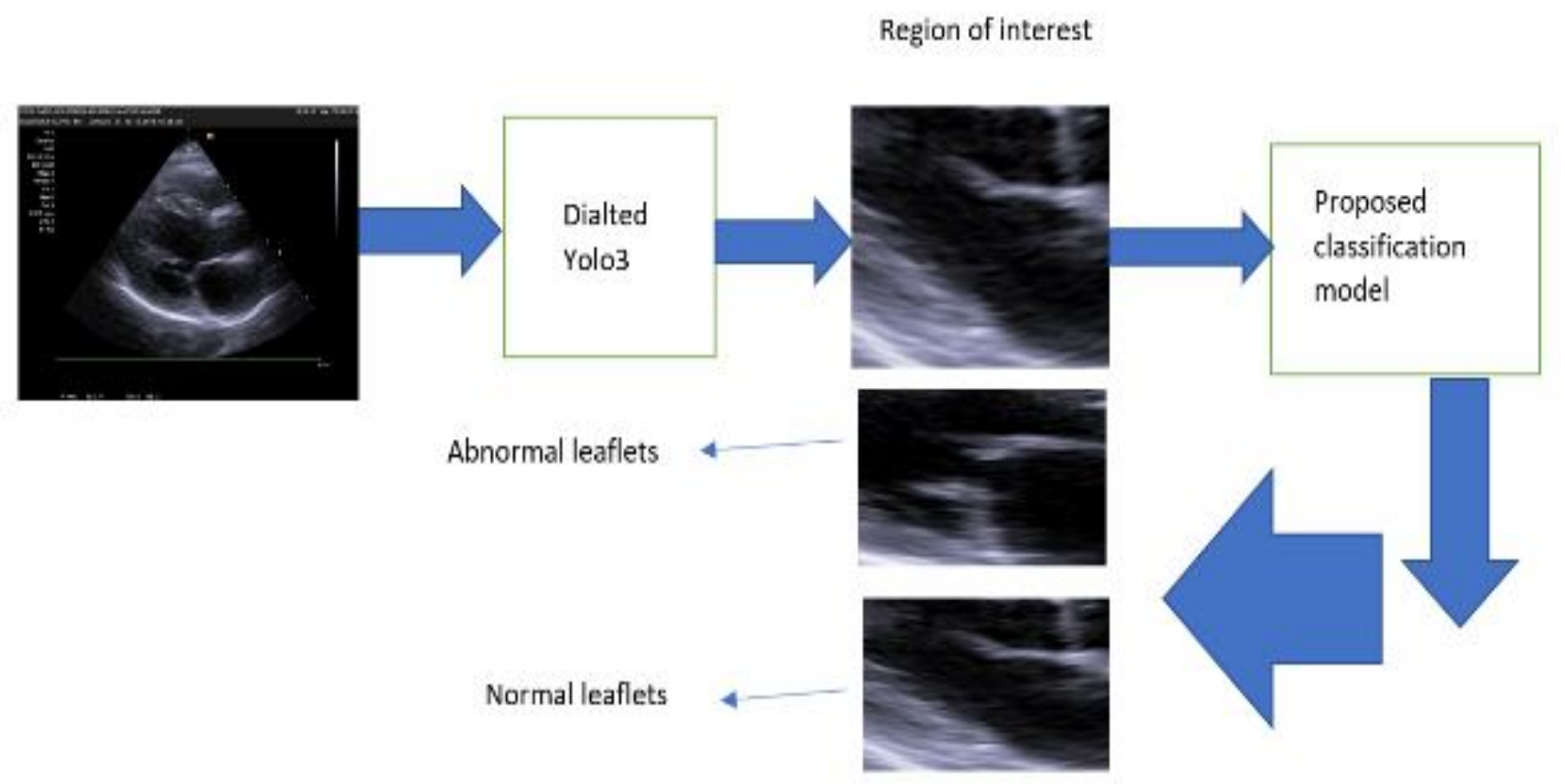

Figure 5:Proposed Pipeline.

Dilated Yolov3 is trained on the collected dataset, it took $12 \mathrm{hr}$ for training, its configurational parameters are shown in table 1. The trained model is used to apply on test datasets for creating a bounding box around mitral leaflets are inside box. The output of dilated yolov3 is automatically cropped to the bounding box and pass as the input to our proposed classifier model. Which gives whether the image of abnormal and normal mitral leaflets. Detection layer which is also last layer is

$\mathrm{DL}=1 \times 1 \times(B \times(5+C))$

$\mathrm{B}=9$ (number of bounding boxes)

$\mathrm{C}=1$ (Mitral Valve)

The configurational of the classification model is shown in table 2. For training of the proposed model took $1 \mathrm{hr}$ with 40 epochs. 
Vishal Chandra et al., International Journal of Emerging Trends in Engineering Research, 8(4), April 2020, 1349 - 1356

Table 1: Configuration of Dilated Yolov3

\begin{tabular}{|l|l|} 
Number of Parameters & $61,576,342$ \\
\hline Image size & \multicolumn{1}{|c|}{$512 \times 512$} \\
\hline Anchors & $\begin{array}{l}{[35,22,24,38,49,87,} \\
67,66,77,24,49,78] \\
\text { using k-Mean } \\
\text { clustering Algorithm }\end{array}$ \\
\hline Maximum box per image & 9 \\
\hline Batch size & 2 \\
\hline Learning rate & $1 \mathrm{e}-4$ \\
\hline Number of epochs & 40 \\
\hline Number of classes & $1($ Mitral Valve $)$ \\
\hline Validation Matrices & mAP \\
\hline
\end{tabular}

Table 2: Configuration of classification model

\begin{tabular}{|c|c|}
\hline $\begin{array}{c}\text { Total Number of } \\
\text { parameters }\end{array}$ & $1,875,649$ \\
\hline Image size & $128 \times 128$ \\
\hline Batch size & 5 \\
\hline Learning rate & $1 \mathrm{e}-4$ \\
\hline Number of epochs & 40 \\
\hline Validation & Class accuracy, F1, \\
\hline Matrices & \\
\hline Optimizer & Adam \\
\hline
\end{tabular}

\section{RESULTS}

After total training time $8 \mathrm{hr} 20 \mathrm{~min}$ model trained without overfitting. Yolov3 has given $86 \mathrm{mAP}$ which is higher. PR(Precision-Recall) curve on testing data for YOLOv3 shown in figure 6 shows the performance of yolov3 to localize mitral leaflets inside bounding boxes.

The performance summary is shown in table 3. As far as the proposed classification model is concerned classification accuracy reached 98.8 percent on test data that never been used in training having F1 score 0.97 and 98 for normal and abnormal leaflets respectively, testing accuracy 99 misclass 0.0147 , ROC curve with AUC 99\%, confusion matrix has drowned, shown in Figures 7 and 8 respectively. The output of classification results shown in figure 9. summary of the classification model is shown in table 3 .



Figure 6: PR Curve of Mitral Valve Dilated YOLOv3

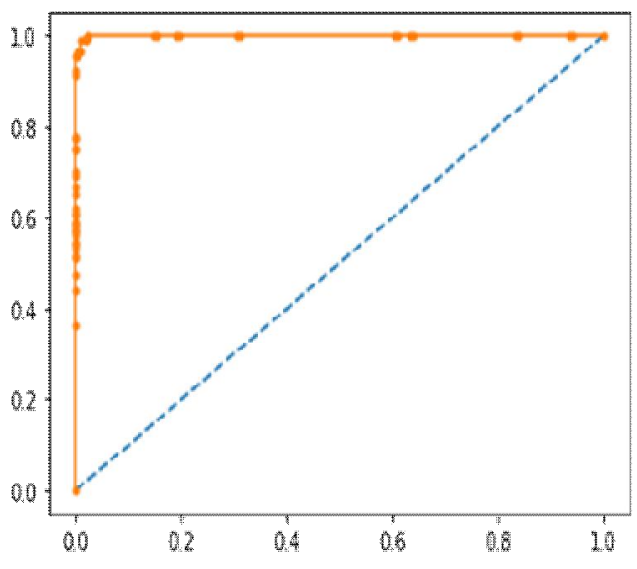

Figure 7:ROC-Curve AUC 0.99

Table 3: Classification Report

\begin{tabular}{|l|l|l|l|l|}
\hline & Precision & Recall & $\begin{array}{l}\text { F1- } \\
\text { score }\end{array}$ & support \\
\hline Normal & 0.99 & 0.98 & 0.97 & 772 \\
\hline Abnormal & 0.99 & 0.99 & 0.98 & 397 \\
\hline Accuracy & & & 0.99 & 1169 \\
\hline $\begin{array}{l}\text { Macro } \\
\text { avg }\end{array}$ & 0.99 & 0.97 & 0.99 & 1169 \\
\hline $\begin{array}{l}\text { Weighted } \\
\text { avg }\end{array}$ & 0.99 & 0.99 & 0.99 & 1169 \\
\hline
\end{tabular}


Vishal Chandra et al., International Journal of Emerging Trends in Engineering Research, 8(4), April 2020, 1349 - 1356

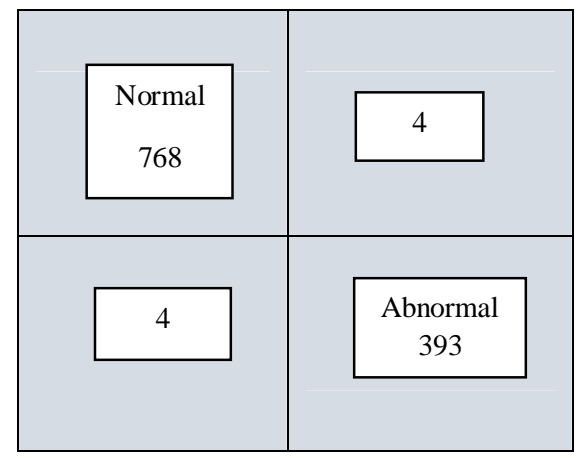

\section{CONCLUSION}

Based on this research we can say deep learning method can assist sonographers and help them not only for diagnosis but also it would take less time per patients. even training with small dataset proposed method gives promising results.

Figure 8 :Confusion Matrix
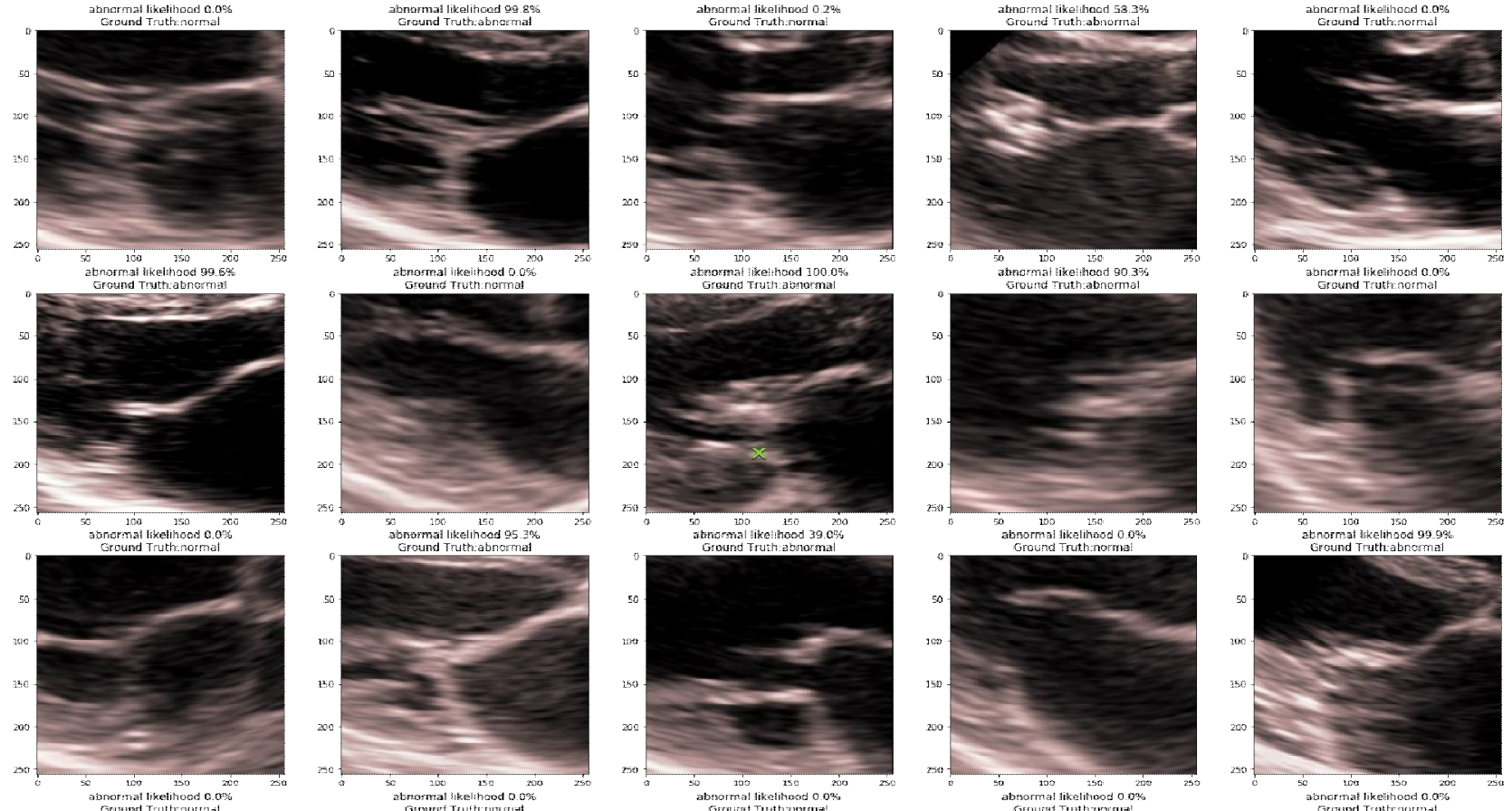




Figure 9: Results 


\section{REFERENCES}

1.S. J. S. B and S. P. Awate, "Hierarchical Generative Modeling and Monte-Carlo EM in Riemannian Shape,"Miccai 2016, vol. 2, pp. 191-200, 2016. https://doi.org/10.1007/978-3-319-46726-9_23

2.A. Barbu, L. Lu, H. Roth, A. Seff, and R. M. Summers, "An analysis of robust cost functions for CNN in computer-aided diagnosis,"Comput. Methods Biomech. Biomed. Eng. Imaging Vis., vol. 6, no. 3, pp. 253-258, 2018.

https://doi.org/10.1080/21681163.2016.1138240

3.S. Bauer, N. Carion, P. Schüffler, T. Fuchs, P. Wild, and J. M. Buhmann, "Multi-Organ Cancer Classification and Survival Analysis," no. Nips, pp. 1-5, 2016.

4.M. R. Berg, "Ingen gevinst av str??lebehandlingvedlivmorkreft," Tidsskr. den Nor. Laegeforening, vol. 133, no. 23-24, p. 2459, 2013. https:// doi.org/ 10.4045/tidsskr.13.1352

5.R. Cervera, "Recent advances in antiphospholipid antibody-related valvulopathies,"J. Autoimmun., vol. 15, no. 2, pp. 123-125, 2000.

6.B. D. de Vos, J. M. Wolterink, P. A. de Jong, M. A. Viergever, and I. Išgum, "2D image classification for $3 \mathrm{D}$ anatomy localization: employing deep convolutional neural networks," Med. Imaging 2016 Image Process., vol. 9784, p. 97841Y, 2016.

https://doi.org/10.1117/12.2216971

7.A. G. Howard et al., "MobileNets: Efficient Convolutional Neural Networks for Mobile Vision Applications," 2017.

8.S. Hwang and H. E. Kim, "Self-transfer learning for weakly supervised lesion localization,'Lect. Notes Comput. Sci. (including Subser. Lect. Notes Artif. Intell. Lect. Notes Bioinformatics), vol. 9901 LNCS, pp. 239246, 2016.

9.E. Marijon, D. S. Celermajer, and X. Jouven, "Rheumatic heart disease - An iceberg in tropical waters,"N. Engl. J. Med., vol. 377, no. 8, pp. 780-781, 2017.

10.N. Navab, J. Hornegger, W. M. Wells, A. F. Frangi, and D. Hutchison, "Medical Image Computing and Computer-Assisted Intervention,” pp. 507-514, 2014.

https://doi.org/10.1007/978-3-319-24553-9

11.J. Redmon and A. Farhadi, "YOLO9000: Better, faster, stronger," Proc. - 30th IEEE Conf. Comput. Vis. Pattern Recognition, CVPR 2017, vol. 2017-Janua, pp. 6517-6525, 2017.

12.J. Redmon and A. Farhadi, "YOLOv3: An Incremental Improvement," 2018.

13.B. Reméanyiet al., "World Heart Federation criteria for echocardiographic diagnosis of rheumatic heart disease-an evidence-based guideline,"Nat. Rev. Cardiol. vol. 9, no. 5, pp. 297-309, 2012.

14.A. Teramoto, H. Fujita, O. Yamamuro, and T. Tamaki, "Automated detection of pulmonary nodules in PET/CT images: Ensemble false-positive reduction using a convolutional neural network technique,"Med. Phys., vol. 43, no. 6, pp. 2821-2827, 2016.

https://doi.org/10.1118/1.4948498

15.M. J. J. P. Van Grinsven, B. Van Ginneken, C. B. Hoyng, T. Theelen, and C. I. Sánchez, "Fast Convolutional Neural Network Training Using Selective Data Sampling: Application to Hemorrhage Detection in Color Fundus Images,'IEEE Trans. Med. Imaging, vol. 35, no. 5, pp. 1273-1284, 2016.

16.D. A. Watkins et al., "Global, regional, \& national burden of rheumatic heart disease, 1990-2015,"

N. Engl. J. Med., vol. 377, no. 8, pp. 713-722, 2017.

17.J. M. Wolterink, T. Leiner, B. D. de Vos, R. W. van Hamersvelt, M. A. Viergever, and I. Išgum, "Automatic coronary artery calcium scoring in cardiac CT angiography using paired convolutional neural networks,"Med. Image Anal., vol. 34, pp. 123-136, 2016. https://doi.org/10.1016/j.media.2016.04.004

18.D. Yang, S. Zhang, Z. Yan, C. Tan, K. Li, and D. Metaxas, "AUTOMATED ANATOMICAL LANDMARK DETECTION ON DISTAL FEMUR SURFACE USING CONVOLUTIONAL NEURAL NETWORK'Department of Computer Science, University of North Carolina at Charlotte, NC, US Department of Industrial and Systems Engineering , Rutgers University,pp. 17-21, 2015.

https://doi.org/10.1109/ISBI.2015.7163806

19.J. Yao, S. Wang, X. Zhu, and J. H. B, "Imaging Biomarker Discovery for Lung Cancer,'Miccai 2016, vol. 1, pp. 649-657, 2016.

20.H. Li et al., "An improved deep learning approach for detection of thyroid papillary cancer in ultrasound images,"Sci. Rep., vol. 8, no. 1, pp. 1-12, 2018.

21.L. Ma, C. Ma, Y. Liu, and X. Wang, "Thyroid diagnosis from SPECT images using convolutional neural network with optimization," Comput. Intell. Neurosci., vol. 2019, 2019. https://doi.org/10.1155/2019/6212759

22.J. Song et al., "Ultrasound image analysis using deep learning algorithm diagnosis of thyroid nodules,"Medicine (Baltimore),, vol. 98, no. 15, p. e15133, 2019.

23.Kusunose K, Abe T, Haga A, Fukuda D, Yamada H, Harada M, et al. A Deep Learning Approach for Assessment of Regional Wall Motion Abnormality From Echocardiographic Images.JACC Cardiovasc Imaging 2019. https://doi.org/10.1016/j.jcmg.2019.02.024. 24.Silva JF, Silva JM, Guerra A, Matos S, Costa C. Ejection Fraction Classification in Transthoracic Echocardiography Using a Deep Learning Approach. Proc - IEEE SympComput Med Syst 2018;2018-June:1238. https://doi.org/10.1109/CBMS.2018.00029.

25.Madani A, Arnaout R, Mofrad M, Arnaout R. Fast and accurate view classification of echocardiograms using deep learning.Npj Digit Med 2018;1:1-8. https://doi.org/10.1038/s41746-017-0013-1.

26. https://github.com/tzutalin/labelImg. 